\title{
LEVANTAMENTO DA AVIFAUNA PRESENTE EM PROPRIEDADE PARTICULAR NO MUNICÍPIO DE SAUDADE DO IGUAÇU, PARANÁ
}

\author{
Bruna Caroline Bratti \\ Fernando Rodrigo Treco
}

BRATTI, B. C.; TRECO, F. R. Levantamento da avifauna presente em propriedade particular do município de Saudade do Iguaçu, Paraná. Arquivos de Ciências Veterinárias e Zoologia da UNIPAR, Umuarama, v. 23, n. 1cont., e2303, 2020.

\begin{abstract}
RESUMO: O Brasil possui uma das mais ricas avifaunas do mundo. Vários estudos com esse grupo vêm sendo realizados na Região Sudoeste do Paraná e também no Brasil. No município de Saudade do Iguaçu não há estudos científicos sobre a comunidade de aves, o que evidencia a importância do presente estudo. Objetivou-se realizar um levantamento da avifauna presente em uma propriedade rural do município de Saudade do Iguaçu, Sudoeste do Paraná. O estudo foi realizado de novembro de 2016 a agosto de 2018. A área de estudo localiza-se em uma propriedade particular na Linha Biguá, que corresponde a 11 hectares; na área predominando a pecuária e a agricultura como atividade econômica e encontram-se fragmentos de mata secundária. Foram realizadas técnicas de observações visuais para identificação das espécies, bem como o registro fotográfico das mesmas. Ao total foram registradas 31 espécies distribuídas em 20 famílias e 10 ordens, as aves com maior frequência de ocorrência (FO) foram Vanellus chilensis (quero-quero) com 43,75\%, e as espécies Furnarius rufus (joão-de-barro) com 46,87\%, Turdus rufiventris (sabiá-laranjeira) com 46,87\%, Sicalis flaveola (canário-da-terra-verdadeiro) com $31,25 \%$ e Cacicus haemorrhous (guaxe) com 34\%. Esses dados contribuem com os demais estudos realizados no estado do Paraná.
\end{abstract}

PALAVRAS-CHAVE: Aves. Brasil. Levantamento.

\section{SURVEY OF BIRD FAUNA ON PRIVATE PROPERTY IN THE CITY OF SAUDADE DO IGUACU, PARANÁ}

\begin{abstract}
Brazil has one of the richest avifauna in the world. Several studies with this group have been carried out in the Southwest Region of Paraná and also in the entire country. Nevertheless, no scientific studies have been performed in the municipality of Saudade do Iguaçu related to its bird community, which highlights the importance of this study. The aim of this study was to survey the bird fauna present in a rural property in the city of Saudade do Iguaçu, in the southwestern region of the state of Paraná. The study was held from November 2016 to August 2018. The study area is located on a private property in the Biguá Line, which corresponds to 11 hectares; the area is exploited predominantly for livestock and agriculture, and it holds fragments of secondary forest. Visual observation techniques were used to identify the species, in addition to photographic record of the species. A total of 31 species were recorded, belonging to 20 families and 10 orders. The birds with the highest prevalence were Vanellus chilensis (Southern lapwig) with 43.75\%; Furnarius rufus (rufous hornero) with 46.87\%; Turdus rufiventris (rufous-bellied thrush) with 46.87\%; Sicalis flaveola (saffron finch) with $31.25 \%$; and Cacicus haemorrhous (red-rumped cacique) with $34 \%$. These data contribute to the other studies carried out in the state of Paraná.
\end{abstract}

KEYWORDS: Birds. Brazil. Survey.

\section{LEVANTAMIENTO DE LA AVIFAUNA PRESENTE EN PROPIEDAD PARTICULAR EN EL MUNICIPIO DE SAUDADE DO IGUAÇU, PARANÁ}

RESUMEN: Brasil posee una de las más ricas avifaunas del mundo. Varios estudios con ese grupo vienen siendo realizados en la Región Suroeste de Paraná y también en Brasil. En el municipio de Saudade do Iguaçu no hay estudios científicos sobre la comunidad de aves, lo que evidencia la importancia del presente estudio. Se objetivó realizar un levantamiento de la avifauna presente en una propiedad rural del municipio de Saudade do Iguaçu, Sudoeste de Paraná. El estudio se realizó de noviembre de 2016 a agosto de 2018. El área de estudio se ubica en una propiedad privada en la Línea Biguá, que corresponde a 11 hectáreas; en el área predominando la ganadería y la agricultura como actividad económica y se encuentran fragmentos de mata secundaria. Se realizaron técnicas de observaciones visuales para identificación de las especies, así como el registro fotográfico de las mismas. En total se registraron 31 especies distribuidas en 20 familias y 10 órdenes, las aves con mayor frecuencia fueron Vanellus chilensis (tero-tero) con el 43,75\%, y las especies Furnarius rufus (hornero) con el 46,87\%, Turdus rufiventris (zorzal colorado) con 46,87\%, Sicalis flaveola (semillero basto) con 31,25\% y Cacicus haemorrhous (boyero cacique) con 34\%. Esos datos contribuyen con los demás estudios realizados en el estado de Paraná. PALABRAS CLAVE: Aves. Brasil. Levantamiento.

DOI: 10.25110 /arqvet.v23i1cont.2020.7277

${ }^{1}$ Acadêmica do curso de Ciências Biológicas da Universidade Paranaense, UNIPAR, Francisco Beltrão, PR.

${ }^{2}$ Professor do curso de Ciências Biológicas da UNIPAR, Francisco Beltrão, PR. 


\section{Introdução}

O Brasil é privilegiado em relação à classe Aves, em seu território há uma grande diversidade podendo ser encontrado desde animais de pequeno porte como beijaflores, até mesmo as grandes aves de rapina como a harpia ou gavião-real (SICK, 2001), além disso o país também se destaca em relação á riqueza desse grupo animal, sendo alocado em segundo lugar no ranking mundial de diversidade, com aproximadamente 1.919 espécies (LUCA et al., 2009; PIACENTINI et al., 2015).

Ao longo dos anos muitas aves vêm sendo domesticadas gerando importância econômica, como é o caso da galinha (Gallus gallus), marrecos, patos e gansos, fornecendo ovos, carne e penas. Algumas pessoas são observadoras das aves e se encantam pelo seu voo, diferentes tipos de cantos e também pelo seu comportamento (SILVEIRA, 2012).

O território brasileiro ocupa quase a metade da América do Sul, possui uma grande variedade de biomas que reflete na grande riqueza de fauna e flora, tendo uma das maiores biodiversidades do planeta, este país também conta com vários ecossistemas na costa marinha (MMA, 2018).

Dentre os biomas nacionais, a Mata Atlântica ocupa um papel de destaque, apresentando uma rica diversidade de aves com aproximadamente 600 espécies, sendo, cerca de 160 endêmicas (WWF, 2010), dados que tornam este ambiente importante em estudos com avifauna.

O bioma da Mata Atlântica ocupa uma área de $1.110 .182 \mathrm{Km}^{2}$, o que corresponde a $13,04 \%$ do território nacional, o estado do Paraná encontra-se nesse bioma. Os estudos com fauna do estado do Paraná iniciaram ainda no século XVI, sendo aos poucos enriquecido pelos naturalistas e cronistas que se estabeleceram. O estado tem grande contribuição com as espécies descritas no país, possui um território de aproximadamente 3,4 milhões de hectares, sua avifauna corresponde a $40 \%$ da avifauna brasileira. (SCHERER NETO et al., 2011; INSTITUTO BRASILEIRO DE FLORESTAS, 2018).

A classe Aves engloba todos os animais vertebrados com presença de penas, membros anteriores modificados em asas e membros posteriores usados para locomoção bipedal (SICK, 2001). Sua anatomia é toda adaptada para voo, suas asas dão sustentação e propulsão. Os ossos devem ser leves e ter uma rígida estrutura. Seu sistema respiratório é eficiente e também é um termorregulador mantendo constante a temperatura corpórea, seu sistema digestório é eficiente e adequado. Seus sentidos são apurados e seu sistema nervoso sintonizado (HICKMAN JUNIOR; ROBERTS; LARSON, 2001).

Os hábitos alimentares estão diretamente relacionados aos diferentes padrões de bicos, podendo existir animais com hábitos frugívoros, que se alimentam de frutos (sanhaçu), granívoros, que se alimentam de grãos (pombas), insetívoros, que se alimentam de insetos (bemte-vi), nectarívoros, que se alimentam de néctar (beija-flor), carnívoros, de carne de animais vivos (gaviões), piscívoros, que se alimentam de peixes (garças), necrófagos, que se alimentam de carne de animais mortos urubu) e onívoros, que são generalistas, com alimentação bastante diversificada (gralha) (SILVEIRA, 2012).
Esses diferentes hábitos estão relacionados às várias contribuições destas espécies para a natureza, algumas são dispersoras de sementes principalmente as aves frugívoras, cujas plantas têm suas sementes dispersas e as aves tem o retorno nutricional. Não somente na dispersão de sementes, as aves atuam na manutenção do equilíbrio ecológico, controle biológico de pragas (insetos, roedores) (FRANCISCO; GALETTI, 2002).

No ano de 1995 o estado do Paraná, contava com 712 espécies diferentes de aves. Em outro estudo realizado em 2011, constavam que o número de espécies observadas era de 744, representando $40 \%$ da fauna brasileira. Mostrando assim, a riqueza ornitológica da fauna paranaense (SCHERER NETO et al., 2011).

Os estudos com a avifauna são importantes porque mostram a diversidade de aves encontradas em determinada região, permite saber se ocorreram mudanças nas populações ao longo dos anos desde o começo dos registros. A Região Sudoeste do Paraná, ainda carece de estudos detalhados sobre a composição da avifauna. Assim, este trabalho tem o objetivo de identificar e registrar a avifauna da propriedade particular no município de Saudade do Iguaçu, Paraná. Tratase do primeiro estudo realizado no município, o que poderá contribuir com o conhecimento da avifauna local.

\section{Material e Métodos}

A presente pesquisa foi realizada entre os meses de novembro de 2016 a agosto de 2018 .

O município de Saudade do Iguaçu se localiza na Região Sudoeste do estado do Paraná, com uma área de 152. $085 \mathrm{~km}^{2}$ (IBGE, 2017). O município encontra-se a 615 metros de altitude do nível do mar, nas coordenadas geográficas: latitude $25^{\circ} 41^{\prime} 13^{\prime}$ ' Sul e longitude $52^{\circ} 36^{\prime} 32$. Com uma população estimada de aproximadamente 5.448 habitantes (CIDADE BRASIL, 2018; IBGE, 2018).

A vegetação predominante no município de Saudade do Iguaçu é da Floresta Ombrófila Mista com transição para Floresta Estacional Semi-decidual (MUCHAILD, 2010).

O presente trabalho foi realizado na Comunidade Linha Biguá em uma propriedade particular que se localiza aproximadamente a $10 \mathrm{~km}$ do centro da cidade.

A área corresponde a 11 hectares, com vegetação de pastagem para gado leiteiro; o gado ocupa quase toda a área, mas não consegue adentrar nos fragmentos de mata presentes. Os fragmentos de mata da propriedade são secundários, possuem árvores nativas e espécies arbóreas exóticas, algumas isoladas, outras nos fragmentos de mata. $\mathrm{Na}$ propriedade encontram-se dois açudes, e o Córrego Creolin.

As saídas a campo foram realizadas quinzenalmente, logo ao amanhecer, período de melhor visualização das aves, com duração de aproximadamente 4 horas.

Foram percorridos trilhas em linha reta ao longo do caminho, com paradas em pontos específicos, locais em que o pesquisador permanecia observando os animais.

As aves foram observadas a olho nu e fotografadas com a câmera de zoom óptico de $42 \mathrm{x}$, para auxiliar na identificação. Para classificação das aves foi utilizado bibliografia específica como Sigrist (2009), Wikiaves (2018).

Foi realizado o cálculo de frequência de ocorrência 
(FO), utilizando-se a seguinte equação:

$F O=\frac{p \times 100}{P}$

Onde p é o número de vezes em que a espécie foi registrada, e $\mathbf{P}$ é o número total de saídas de campo realizadas. A curva do coletor é acumulativa de espécies.

Os hábitos alimentares foram classificados em frugívoras, granívoros, insetívoros, nectarívoros, carnívoros, piscívoros, necrófagos e onívoros considerando o principal alimento da espécie, sendo usadas literaturas como Sick (2001), Sigrist (2009) e Wikiaves (2018).

Para a classificação das espécies como residentes ou migratórias foi avaliado informações da literatura (SIGRIS,T 2013; WIKIAVES, 2018) em associação com a FO, uma vez que as espécies migratórias são encontradas em épocas específicas ao decorrer do ano.

\section{Resultados e Discussão}

Com esforço amostral de 136 horas, foi possível identificar 31 espécies distribuídas em 20 famílias e 10 ordens (Tabela 1). O esforço amostral se demonstrou suficiente, uma vez que a curva do coletor permaneceu estável nas últimas dez saídas a campo (Figura 1).

Tabela 1: Aves identificadas em propriedade rural no município de Saudade do Iguaçu, Paraná.

\begin{tabular}{|c|c|c|c|c|c|c|}
\hline Ordem & Família & Espécie & Nome popular & FO & MI & so \\
\hline Charadriiformes & Charadriidae & Vanellus chilensis & quero-quero & $43,75 \%$ & $\mathrm{OF}$ & $\mathrm{R}$ \\
\hline \multirow[t]{4}{*}{ Columbiformes } & Columbidae & Columbina squammata & fogo-apagou & $3,12 \%$ & OF & $\mathrm{R}$ \\
\hline & & Columbina talpacoti & rolinha-roxa & $9,37 \%$ & OF & $\mathrm{R}$ \\
\hline & & Columbina minuta & $\begin{array}{l}\text { rolinha-asa- } \\
\text { canela }\end{array}$ & $12,50 \%$ & OF & M \\
\hline & & Patagioenas picazuro & $\begin{array}{l}\text { pombão/pomba- } \\
\text { cariió }\end{array}$ & $3,12 \%$ & OF & M \\
\hline Coraciiformes & Momotidae & $\begin{array}{l}\text { Baryphthengus } \\
\text { ruficapillus }\end{array}$ & juruva-verde & $3,12 \%$ & $\mathrm{OF}$ & $\mathrm{R}$ \\
\hline Cuculiformes & Cuculidae & Piaya cayana & alma-de-gato & $15,62 \%$ & OF & $\mathrm{R}$ \\
\hline Galliformes & Cracidae & Penelope obscura & jacu/jacuaçu & $3,12 \%$ & OF & $\mathrm{R}$ \\
\hline \multirow[t]{15}{*}{ Passeriformes } & Corvidae & Cyanocorax chrysops & gralha-picaça & $25 \%$ & OF & $\mathrm{R}$ \\
\hline & Dendrocolaptidae & $\begin{array}{l}\text { Dendrocolaptes } \\
\text { platyrostris }\end{array}$ & arapaçu-grande & $3,12 \%$ & OF & $\mathrm{R}$ \\
\hline & Furnariidae & Furnarius rufus & joão-de-barro & $46,87 \%$ & OF & $\mathrm{R}$ \\
\hline & Icteridae & Cacicus haemorrhous & guaxe & $34 \%$ & OF & $\mathrm{R}$ \\
\hline & Thraupidae & Sicalis flaveola & $\begin{array}{l}\text { canário-da-terra- } \\
\text { verdadeiro }\end{array}$ & $31,25 \%$ & OF & $\mathrm{R}$ \\
\hline & & $\begin{array}{l}\text { Coryphospingus } \\
\text { cucullatus }\end{array}$ & tico-tico-rei & $3,12 \%$ & OF & M \\
\hline & & Tangara sayaca & sanhaçu-cinzento & $25 \%$ & OF & $\mathrm{R}$ \\
\hline & & Cissopis leverianus & tietinga & $3,12 \%$ & OF & $\mathrm{R}$ \\
\hline & Troglodytidae & Troglodytes musculus & corruíra-de-casa & $3,12 \%$ & $\mathrm{OF}$ & $\mathrm{R}$ \\
\hline & & Turdus rufiventris & sabiá-laranjeira & $46,87 \%$ & OF & $\mathrm{R}$ \\
\hline & Tyrannidae & $\begin{array}{l}\text { Myiodynastes } \\
\text { maculatus }\end{array}$ & bem-te-vi-rajado & $9,30 \%$ & $\mathrm{OF}$ & M \\
\hline & & Pitangus sulphuratus & bem-te-vi & $25 \%$ & OF & M \\
\hline & & $\begin{array}{l}\text { Tyrannus } \\
\text { melancholicus }\end{array}$ & Suiriri & $3,12 \%$ & OF & M \\
\hline & & Tyrannus savana & tesourinha & $3,12 \%$ & OF & M \\
\hline & & Zonotrichia capensis & tico-tico & $12,50 \%$ & OF & $\mathrm{R}$ \\
\hline \multirow[t]{2}{*}{ Pelecaniformes } & Ardeidae & Bubulcus ibis & garça-vaqueira & $15,62 \%$ & OF & M \\
\hline & Threskiornithidae & Theristicus caudatus & curicaca & $6,25 \%$ & $\mathrm{OF}$ & $\mathrm{R}$ \\
\hline \multirow[t]{3}{*}{ Piciformes } & Picidae & Colaptes campestris & $\begin{array}{l}\text { pica-pau-do- } \\
\text { campo }\end{array}$ & $3,12 \%$ & OF & M \\
\hline & & $\begin{array}{l}\text { Colaptes } \\
\text { melanochloros }\end{array}$ & $\begin{array}{l}\text { pica-pau-verde- } \\
\text { barrado }\end{array}$ & $6,25 \%$ & OF & M \\
\hline & Ramphastidae & Ramphastos dicolorus & $\begin{array}{l}\text { tucano-de bico- } \\
\text { verde }\end{array}$ & $6,25 \%$ & OF & M \\
\hline Psittaciformes & Psittacidae & Pyrrhura frontalis & $\begin{array}{l}\text { tiriba-de-testa- } \\
\text { vermelha }\end{array}$ & $3,12 \%$ & $\mathrm{OF}$ & $\mathrm{R}$ \\
\hline Trogoniformes & Trogonidae & Trogon surrucura & surucuá-variado & $6,25 \%$ & OF & $\mathrm{M}$ \\
\hline
\end{tabular}

Registros: métodos de identificação (MI), observada (O), fotografada $(\mathrm{F})$, status de ocorrência $(\mathrm{SO})$ migratória $(\mathrm{M})$ e residente (R).
Figura 1: Curva do coletor referente às espécies de aves registradas nas saídas de campo realizadas na propriedade do município de Saudade do Iguaçu-Paraná (onde c1, c2 e assim sucessivamente representam as espécies).

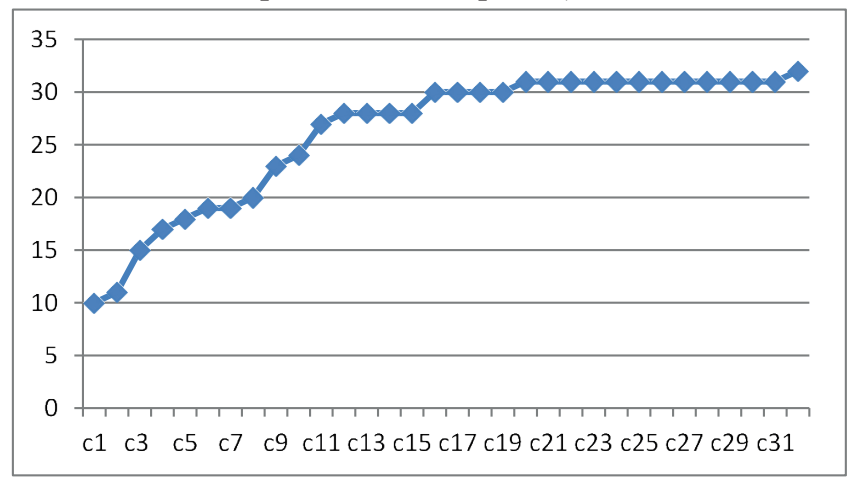

As espécies pertencentes a Ordem Passeriformes foram as mais visualizadas (Tabela 1), tal grupo compõe a maior ordem de aves do mundo, para o país representam $55,52 \%$ das espécies catalogadas (MARINI; GARCIA, 2005), característica que permite uma maior visualização das mesmas. Além disso, a dieta dos passeriformes é muito variada, e possuem uma ampla distribuição geográfica, não ocorrendo apenas no continente antártico (SILVEIRA, 2012), fatores que permitem uma grande adaptabilidade a diferentes ambientes do planeta.

As aves que obtiveram maior frequência de ocorrência foram as espécies Vanellus chilensis (quero-quero) com 43,75\%, Furnarius rufus (joão-de-barro) com 46,87\%, Turdus rufiventris (sabiá-laranjeira) com 46,87\%, Sicalis flaveola (canário-da-terra-verdadeiro) com $31,25 \%$ e Cacicus haemorrhous (guaxe) com 34\% (Tabela 1). Tais espécies são bastante comuns em ambientes antropizados, constituem um grupo de aves com hábito alimentar generalista, o que proporciona um melhor acesso ao alimento ao decorrer do ano, ademais, a área de estudo destaca-se atividade agropecuária, prática que produz resíduos alimentares que frequentemente atraem estes animais. Tal característica também relatada no município do Verê, PR, ambiente próximo à área estudada (ESSY; TRECO, 2015).

Das cinco espécies que obtiveram maior frequência de ocorrência, quatro delas pertencem à Ordem Passeriformes, apenas com exceção do Vanellus chilensis. Resultado semelhante ao de Torga, Franchin e Marçal Junior (2004) em Uberlândia, Minas Gerais, Mencato e Treco (2016) em Verê, Paraná, Essy e Treco (2015) em Verê, Paraná, Schafhauser, Richter e Portela (2009) no município de Pinhais, Paraná, onde a Ordem Passeriformes teve maior frequência de ocorrência. Esses resultados demonstram a alta prevalência dos passeriformes na região Sudoeste do Paraná e em outras regiões do país.

Vale ressaltar que a frequência de ocorrência da comunidade estudada não se demonstrou alta quando comparada a outros estudos, esse resultado demonstra forte influência da fragmentação, característica da área estudada, onde a comunidade de aves é reduzida devido ao tamanho e quantidade dos fragmentos. De acordo com Mohr (2012), a presença de fragmentação, em decorrência da alteração de paisagem natural, leva a uma dificuldade de deslocamento dos animais entre as áreas fragmentadas, o que afeta diretamente 
o tamanho populacional das mesmas.

Ademais, a formação de fragmentos em uma área implica na formação de uma borda florestal, que é a região em contato com a área ocupada e o fragmento de vegetação natural. Isso gera alterações físicas, químicas e biológicas na área fazendo com que muitas espécies não consigam seu alimento levando a migração para outro fragmento ou até mesmo afetando na reprodução, impedindo a perpetuação destas espécies (PRIMAK; RODRIGUES, 2001).

Em relação aos hábitos alimentares (Figura 2) destacam-se espécies de hábitos onívoros (40\%) espécies de hábitos insetívoros $(30 \%)$, resultados semelhantes ao de Scherer, Scherer e Petry (2010) em Porto Alegre-RS; onde ambas as áreas de estudo, existe ação humana. As espécies com hábito alimentar onívoro se destacam, pois são espécies cuja alimentação é generalista tendo oferta de alimento o ano todo (SCHERER; SCHERER; PETRY, 2010). Espécies com esse tipo de hábito alimentar mostram que em ambientes antropizados, como é o caso da área estudada, se sobressai às demais espécies com outros hábitos, pois, elas encontram seu alimento com facilidade.

Figura 2: Hábitos alimentares correspondentes as 31 espécies de aves identificadas no município Saudade do Iguaçu-Paraná.

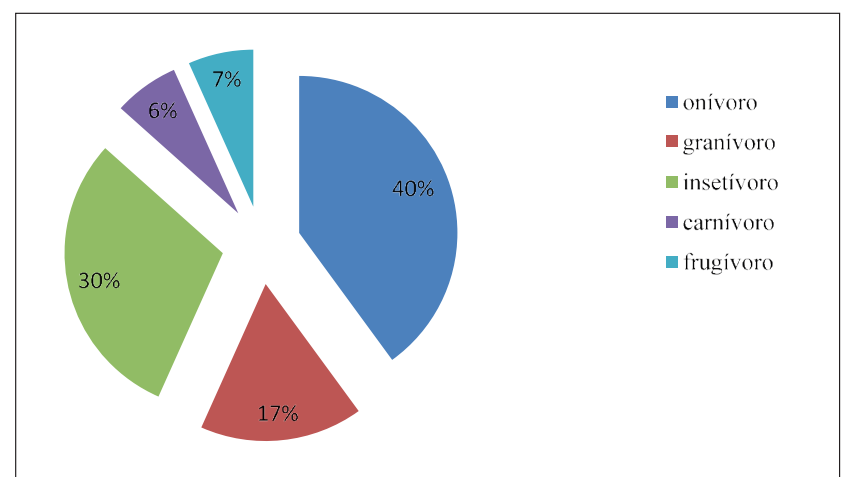

\section{Conclusão}

Esse estudo demonstra grande importância, pois é o primeiro estudo com esses animais no município mostrando a diversidade de aves que habita a região.

A avifauna da propriedade rural de Saudade do Iguaçu Paraná é composta por 31 espécies distribuídas em 20 famílias e 10 ordens. As espécies registradas que obtiveram a maior frequência adaptaram-se de alguma forma ao ambiente antropizado, encontrando assim condições para sua sobrevivência.

Em relação aos hábitos alimentares destacam-se as espécies onívoras e insetívoras, pela disponibilidade e facilidade de encontrar alimento no decorrer do ano.

\section{Referências}

CIDADE BRASIL. Município de Saudade do Iguaçu. Disponível em: http://m.cidade-brasil.com.br. Acesso em: 28 fev. 2018.

ESSY, A. B. C.; TRECO, F. R. Comunidade de aves urbanas do município de Verê- Paraná. Arquivos de Ciências Veterinárias e Zoologia da UNIPAR, Umuarama, v. 18, n. 3, p. 169-174, 2015.

FRANCISCO, M. R.; GALETTI, M. Aves como potenciais dispersoras de sementes de Ocotea pulchella Mart.

(Lauraceae) numa área de vegetação de cerrado do sudeste brasileiro. Revista Brasileira de Botânica, São Paulo, v. 25, n. 1, p. 11-17, 2002.

HICKMAN JR., P. C.; ROBERTS, S. L; LARSON, A. Princípios Integrados de Zoologia. Rio de Janeiro: Guanabara, p. 846, 2001.

IBGE. Instituto Brasileiro de Geografia e Estatística. Disponível em: http://cidades.ibge.org.br. Acesso em: 28 fev. 2018 .

\section{INSTITUTO BRASILEIRO DE FLORESTAS. Bioma}

Mata Atlântica. Disponível em: http://www.ibflorestas.org. br. Acesso em: 02 mar. 2018.

LUCA, C. A. et al. Aves da Mata Atlântica do Nordeste. Save Brasil. São Paulo, dezembro de 2009.

MARINI, A. M.; GARCIA, L. F. Conservação de aves no Brasil. Revista Megadiversidade, v. 1, n. 1, p. 95-102 jul., 2005.

MENCATO, A. A.; TRECO, F. R. Estrutura e composição da avifauna em um ambiente rural no sul do Brasil. Revista Ciência, Tecnologia e Ambiente, v. 3, n. 1, p. 12-20, 2016.

MOHR, S. R. L. Distribuição da avifauna em ambientes fragmentados de Floresta Ombrófila Mista na bacia hidrográfica do Rio Forqueta, RS, Brasil. Centro Universitário UNIVATEs, Ambiente e Desenvolvimento Programa de pós-graduação. Lajeado, 2012.

MMA. Ministério do Meio Ambiente. Espécies exóticas invasoras. Disponível em: http://www.mma.gov.br. Acesso em: 02 mar. 2018

MUCHAILD, C. M. Metodologia de Planejamento da Paisagem para Sustentabilidade Ambiental Região Centro Sul do Paraná. Universidade Federal do Paraná, Setor de Ciências Agrárias. Curitiba, 2010.

PIACENTINI, V. Q. et al. Annotated checklist of the birds of Brazil by the Brazilian Ornithological Records Committee / Lista comentada das aves do Brasil pelo Comitê Brasileiro de Registros Ornitológicos. Revista Brasileira de Ornitologia, v. 23, n. 2, p. 91-298, 2015.

PRIMACK, R. B.; RODRIGUES, E. Biologia da Conservação. Londrina, 2001.

SCHAFHAUSER, E.; RICHTER, A. S.; PORTELA, G. S. Estudo da Avifauna Nativa como Ferramenta Complementar de Diagnóstico Ambiental. Revista Brasileira de Agroecologia, v. 4, n. 2, dec. 2009. 
SCHERER NETO, P. et al. Lista de Aves do Paraná. 1. ed. Curitiba: Hori Consultoria Ambiental. Hori Cadernos Técnicos n. 2, p. 1362011.

SCHERER, J. F. M.; SCHERER, A. L.; PETRY, M. V. Estrutura trófica e ocupação de hábitat da avifauna de um parque urbano em Porto Alegre, Rio Grande do Sul, Brasil. Revista Biotemas, v. 23, n. 1, p. 169-180, 2010.

SICK, H. Ornitologia Brasileira. Rio de Janeiro: Nova Fronteira, p. 862, 2001.

SIGRIST, E. Guia de campo avis brasilis Avifauna Brasileira. São Paulo: Avis Brasilis, p. 480, 2009.

SILVEIRA, F. L. Universidade de São Paulo-Apostila Ornitologia Básica. São Paulo, 2012.

TORGA, K.; FRANCHIN, A. G.; MARÇAL JÚNIOR, O. A avifauna em uma seção da área urbana de Uberlândia, MG. Revista Biotemas, v. 20, n. 1, p. 7-17, 2007.

WIKIAVES. Enciclopédia de aves do Brasil. Disponível em: http://www.wikiaves.com.br. Acesso em: 02 mar.2018.

WWF. World Wide Fund For Nature. Disponível em: http://www.wwf.org.br. Acesso em: 02 mar. 2018.

Recebido em: 17/12/2018

Aceito em: 01/06/2020 